\title{
Hitting the tuberculosis wall
}

Rising resistance rates to current anti-tuberculosis drugs highlight the need for new drugs that have novel mechanisms of action. A trio of recent papers suggests that enzymes involved in mycobacterial cell wall synthesis could be attractive drug targets.

The papers by Wilson et al. and Stanley et al. identified antituberculosis compounds that inhibit two new targets - PKS13 and FadD32 - in the mycolic acid biosynthesis pathway. This pathway is essential for cell wall synthesis and is where the established antituberculosis drug isoniazid acts. Specifically, FadD32 is a long-chain fatty acyl-AMP ligase that adenylates a long-chain fatty acid and then transfers this activated intermediate to the polyketide synthase PKS13, which then joins the adenylated fatty acid chain to another fatty acid.

Wilson et al. screened small molecules with known activity against Mycobacterium tuberculosis to identify compounds that induced the expression of a gene cluster (piniBAC) to select for inhibitors of cell wall biosynthesis. Two thiophene compounds (termed TP2 and TP4) from this screen that were active against drug-susceptible and multidrug-resistant $M$. tuberculosis strains (minimum inhibitory concentration (MIC): 0.5 to $1.0 \mu \mathrm{M}$ ) were investigated further.

The authors then determined that the compounds targeted PKS13, specifically by inhibiting FadD32dependent loading of fatty acyl-AMP onto the acyl carrier protein of PKS13. Indeed, computational docking studies identified a potential binding groove for the thiophene compounds within the acyl carrier protein domain of PKS13.

In vitro, TP2 and TP4 killed actively growing $M$. tuberculosis and, notably, the compounds were less likely to promote resistance than isoniazid. Furthermore, combined isoniazid and TP2 or TP4 treatment sterilized the cell cultures.

Stanley et al. screened compounds for their ability to inhibit the growth of $M$. tuberculosis. Optimization of hit compounds that were characterized by the presence of a 4,6-diaryl5,7-dimethyl coumarin core resulted in a compound (termed CCA34; MIC: $0.24 \mu \mathrm{M}$ ) that was shown to inhibit the acyl carrier protein synthetase activity of FadD32.

Studies in zebrafish embryos infected with Mycobacterium marinum showed that CCA34 penetrated host tissues and inhibited proliferation of bacilli. In mice infected with M. tuberculosis, 8-day treatment with CCA34 (administered by injection beginning 1 week after infection) reduced bacterial levels in the lungs of treated mice - an effect that was comparable to that of isoniazid.
The study by Wang et al. identified TCA1 as an inhibitor of DprE1 (decaprenylphosphoryl- $\beta$-D-ribose 2 '-epimerase 1 ), a previously identified anti-tuberculosis target involved in cell wall synthesis. The compound also inhibited MoeW, an enzyme that is involved in the biosynthesis of the molybdenum cofactor. TCA1 was identified from a screen of heterocycle compounds for its ability to inhibit biofilm formation in Mycobacterium smegmatis.

TCA1 had bactericidal activity against drug-susceptible and multidrug-resistant $M$. tuberculosis strains. When TCA1 was combined with rifampicin or isoniazid, it sterilized cell cultures and also had bactericidal activity against non-replicating $M$. tuberculosis.

In a mouse model of chronic M. tuberculosis infection, treatment with TCA1 (given by injection beginning 4 weeks after infection) for 4 weeks reduced infection in lungs and spleen, and showed synergistic actions when combined with isoniazid.

Although further optimization of the compounds identified in these studies is needed (for example, their potency and drug-like properties), they open the door to new ways of targeting tuberculosis.

Charlotte Harrison

ORIGINAL RESEARCH PAPERS Stanley, S. A.

et al. Diarylcoumarins inhibit mycolic acid biosynthesis and kill Mycobacterium tuberculosis by targeting FadD32. Proc. Natl Acad. Sci. USA 110, 11565-11570 (2013) |Wilson, R. et al. Antituberculosis thiophenes define a requirement for Pks13 in mycolic acid biosynthesis

Nature Chem. Biol. http://dx.doi.org/10.1038/ nchembio.1277 (2013) |Wang, F. et al. Identification of a small molecule with activity against drug-resistant and persistent tuberculosis. Proc. Natl Acad. Sci. USA 110, E2510-E2517 (2013) 